\title{
Restless leg syndrome in children with celiac disease
}

\author{
Sedat Işıkay ${ }^{1}$, Nurgül Işıkay², Hüseyin Per ${ }^{3}$, Kürşat Bora Çarman ${ }^{4}$, Halil Kocamaz ${ }^{5}$ \\ ${ }^{1}$ Department of Physiotherapy and Rehabilitation, Hasan Kalyoncu University, School of Health Sciences, Gaziantep; \\ ${ }^{2}$ Department of Anesthesiology, Şehitkamil Goverment Hospital, Gaziantep; ${ }^{3}$ Department of Pediatric Neurology, Erciyes \\ University, Faculty of Medicine, Kayseri; ${ }^{4}$ Department of Pediatric Neurology, Eskisehir Osmangazi University, Faculty \\ of Medicine, Eskisehir; ${ }^{5}$ Department of Pediatric Gastroenterology, Pamukkale University, Faculty of Medicine, Denizli, \\ Turkey.E-mail: dr.sedatisikay@mynet.com
}

Received: 7th December 2016, Revised: 15th February 2017, Accepted: 20th February 2017

SUMMARY: Işıkay S, Işıkay N, Per H, Çarman KB, Kocamaz H. Restless leg syndrome in children with celiac disease. Turk J Pediatr 2018; 60: 70-75.

Celiac disease (CD) is an immune-mediated enteropathy triggered by ingestion of dietary gluten in genetically predisposed individuals. The aim of the study was to determine the prevalence of restless leg syndrome (RLS) in children with CD and to investigate the associated factors for RLS. Totally 494 children with the ages ranging between 11-18 years were included. Among those, 226 were under follow-up with $C D$ and constituted the study group while other 268 children did not have any symptoms or signs associated with CD and established the control group. The demographic data, educational status and routine laboratory data of children including complete blood count, ferritin, vitamin $\mathrm{B} 12$, foliate and $25(\mathrm{OH})$ vitamin D levels were recorded. The RLS prevalence and associated symptoms of children were defined with a questionnaire. There was no statistically significant difference between the 2 groups regarding the age and gender. Moreover, RLS prevalence was also similar in both groups $(3.5 \%$ vs $3.0 \%$ in $\mathrm{CD}$ and control groups, respectively, $\mathrm{p}=0.98$ ). However, interestingly, in $\mathrm{CD}$ group, the mean age of the patients at the onset of RLS symptoms was statistically significantly younger $(p=0.02)$ and the disease was more severe $(p=0.026)$ than the control group. In correlation analysis in CD group, the RLS severity significantly negatively correlated with serum ferritin, folic acid or $25(\mathrm{OH})$ vitamin D levels in Celiac disease group. In this study we did not determine an increased prevalence of RLS in children with CD. However, in CD group, the age at the onset of RLS symptoms was significantly younger and the disease was more severe in CD group compared with the control cases.

Key words: celiac disease, restless leg syndrome, children, anemia.

Celiac disease (CD) is an immune-mediated enteropathy triggered by ingestion of dietary gluten in genetically predisposed individuals. ${ }^{1}$ In clinical practice $C D$ is clearly related to malabsorption, iron deficiency, osteoporosis, and an increased risk of lymphoma as well as extra-intestinal symptoms especially associated with autoimmune diseases including diabetes mellitus type 1 and thyroiditis. ${ }^{2,3}$

Restless legs syndrome (RLS), is a sensorimotor defect which consists of a discomfort in one's legs causing an irresistible desire to move them most commonly occurring in the evening or at night. ${ }^{4}$ The symptoms generally disappear with movement but get worse during rest periods. ${ }^{5}$
RLS is a common clinical syndrome effecting about $5-15 \%$ of the general population. 6,7 Although the dopaminergic pathways and iron metabolism in the substantia nigra has been accused; the pathogenesis of RLS still could not be elucidated clearly. ${ }^{8,9}$ Regarding the immunogenic pathway and/or iron deficiency anemia as causative factors, RLS may be related with CD.

In literature, the data about the association of RLS with CD is limited. In this study, we aimed to determine the prevalence of RLS in children with $\mathrm{CD}$ and to investigate the associated factors for RLS such as iron and vitamin levels. 


\section{Material and Methods}

\section{Study participants}

This cross-sectional study was performed at the Department of Pediatrics, Sütçü İmam University Hospital, Kahramanmaraş, Turkey between March 2015 and June 2016. Totally 494 children with the ages ranging between 11-18 years living in Gaziantep and Kahramanmaraş were included. Among those, 226 were under follow-up for $\mathrm{CD}$ and constituted the study group while the other 268 children without any symptoms or signs associated with $C D$ established the control group. The patients of the control group were selected from the patients who were admitted to our hospital for acute diseases such as upper airway infection and who were not diagnosed with any chronic diseases before. The diagnosis of CD was based on serum positivity for EMA IgA and/or antitissue transglutaminase and on histopathological findings of the intestinal mucosa. The patients with CD were classified according to the Marsh classification system. ${ }^{10,11}$ All celiac patients were under gluten free diet (GFD) but for different time periods (3 months-5 years).

Informed consent was obtained from the parents of all children and the study was approved by the local ethics committee. There was no patient previously diagnosed with RLS who was included in or excluded from the study.

The demographic data, educational status and routine laboratory data of children including complete blood count, ferritin, vitamin B12, folate and $25(\mathrm{OH})$ vitamin D levels were recorded. The RLS prevalence and associated symptoms of children were defined with a 30-questioned questionnaire. The RLS was diagnosed according to the International RLS Study Group (IRLSSG) diagnostic criteria and symptom severity was evaluated using the IRLSSG rating scale. ${ }^{12}$

\section{Statistical analysis}

Statistical analyses of the data were performed with the SPSS program (Statistical Package for the Social Sciences version 21, Chicago, IL, USA). The quantitative variables were described as the mean $\pm \mathrm{SD}$ and the categorical variables as the frequency and percentage. Comparisons of demographic features and biochemical data of groups were performed with student $t$ test. Pearson correlation analysis was performed to determine the association of biochemical data with the age at the onset of RLS symptoms and disease severity in $\mathrm{CD}$ group. A P value $<0.05$ was considered statistically significant.

\section{Results}

Totally 494 children, 226 in CD group with a mean age of $13.20 \pm 2.62$ years and 268 in control group with a mean age of $13.62 \pm 1.02$ years, were included in this study. There was not any statistically significant difference between 2 groups regarding the age, gender and educational level (Table I). Moreover, RLS prevalence was also similar in both groups (3.5\% vs $3.0 \%$ in CD and control groups, respectively, $\mathrm{p}=0.98)$. However, interestingly,

Table I. The General Characteristics and Laboratory Data of Subjects Included in The Study.

\begin{tabular}{lccc}
\hline & $\begin{array}{c}\text { Celiac disease group } \\
(\mathrm{n}=226)\end{array}$ & $\begin{array}{c}\text { Control group } \\
(\mathrm{n}=268)\end{array}$ & $\mathrm{p}$ \\
\hline Age (year) & $13.20 \pm 2.62$ & $13.62 \pm 1.02$ & 0.11 \\
Gender (Female/Male) & $106 / 120$ & $140 / 128$ & 0.44 \\
Average course grade & $75.56 \pm 14.69$ & $86.83 \pm 61.58$ & 0.09 \\
RLS prevalence n (\%) & $8(3.5)$ & $8(3.0)$ & 0.98 \\
The mean age at the onset of RLS & $9.30 \pm 3.53$ & $12.00 \pm 2.09$ & 0.002 \\
symptoms (years) & $12.18 \pm 1.17$ & $12.35 \pm 0.96$ & 0.61 \\
Hemoglobin (g/dl) & $16.48 \pm 12.57$ & $24.11 \pm 5.78$ & 0.04 \\
Ferritin (ng/ml) & $350.72 \pm 137.04$ & $327.55 \pm 124.32$ & 0.62 \\
Vitamin B12 (pg/ml) & $12.55 \pm 18.04$ & $16.50 \pm 12.14$ & 0.54 \\
Folic acid (ng/ml) & $12.39 \pm 12.80$ & $14.33 \pm 3.80$ & 0.65 \\
25 (OH) vitamin D & & & \\
\hline
\end{tabular}

RLS: restless leg syndrome 
in CD group, the mean age of the patients at the onset of RLS symptoms was statistically significantly younger than the control group $(p=0.02)$. The general characteristics and laboratory data of subjects included in the study are summarized in Table I.

When the patients diagnosed with RLS were sub-grouped as mild, moderate or severe; in the CD group among 8 RLS cases; 2 were mild and 6 were in severe group while in control group among 8 RLS cases; 4 were mild and 4 were in moderate group and there was a statistically significant difference between 2 groups regarding the severity of RLS $(p=0.026)$. All celiac patients diagnosed with RLS had Marsh type $3 a(n=2)$ or $3 b(n=2)$ in duodenal biopsy. When celiac patients were sub-grouped regarding the presence or absence of RLS, and those 2 groups were compared for the laboratory data (Table II); serum ferritin and $25(\mathrm{OH})$ vitamin D levels were statistically significantly lower in patients diagnosed with RLS.

In correlation analysis in CD group, there was not any statistically significant association between the age of the patients at the onset of RLS symptoms and serum hemoglobin, ferritin, Vitamin B12, folic acid and $25(\mathrm{OH})$ vitamin D levels (Table III). However, the RLS severity significantly negatively correlated with serum ferritin, folic acid or $25(\mathrm{OH})$ vitamin $\mathrm{D}$ levels in CD group.

\section{Discussion}

In this study, we have investigated the RLS prevalence and associated factors in children with CD and did not determine any statistically significant difference between CD patients and control group regarding the RLS prevalence. However, interestingly, the onset of symptoms of RLS was reported at a younger age and the disease was more severe in $\mathrm{CD}$ group compared with the control cases. Moreover, the celiac patients with RLS all had received Marsh 3 type of CD diagnosis on duodenal biopsy and had significantly lower serum ferritin and 25 $(\mathrm{OH})$ vitamin $\mathrm{D}$ levels compared with the $\mathrm{CD}$ patients without RLS. Additionally, the RLS severity significantly negatively correlated with serum ferritin, folic acid and $25(\mathrm{OH})$ vitamin $\mathrm{D}$ levels in $\mathrm{CD}$ group.

The data about the association of RLS with CD is limited in literature. Moccia et al. ${ }^{13}$ reported RLS prevalence as high as $31 \%$ in 100 adult CD patients which was significantly higher than the prevalence in the control population (4\%). Moreover, they defined the average severity of RLS in CD population as moderate and they did not define any significant correlation between RLS and either gluten-free diet (GFD) or iron metabolism. Weinstock et al. ${ }^{14}$ reported the prevalence of RLS as $25 \%$ in 85 CD patients which was statistically significantly higher than that of their spouses (10\%). They reported that, after GFD, the RLS symptoms improved in $50 \%$ of patients. On the other hand, Marild et al. ${ }^{15}$ investigated the sleep disturbances among patients with CD in a nationwide casecontrol study and reported the RLS prevalence as $0.6 \%$ both in CD group $(19 / 2933)$ and in the controls (89/14 571). Cirikcioglu et al. ${ }^{16}$ investigated 96 RLS patients and 97 healthy controls for the presence of $\mathrm{CD}$ antibodies and reported that the prevalence of these antibodies was not significantly different between the 2 groups. In this study, we have determined the

Table II. Comparison of Celiac Patients With or Without RLS.

\begin{tabular}{lccc}
\hline & $\begin{array}{c}\text { Celiac patients without RLS } \\
(\mathrm{n}=218)\end{array}$ & $\begin{array}{c}\text { Celiac patients with RLS } \\
(\mathrm{n}=8)\end{array}$ & $\mathrm{p}$ \\
\hline Age (year) & $13.24 \pm 2.42$ & $13.12 \pm 0.84$ & 0.92 \\
Gender (Female/Male) & $101 / 117$ & $5 / 3$ & 0.68 \\
Average course grade & $76.18 \pm 12.74$ & $72.83 \pm 14.68$ & 0.44 \\
Hemoglobin $(\mathrm{g} / \mathrm{dl})$ & $12.12 \pm 1.16$ & $12.26 \pm 0.88$ & 0.88 \\
Ferritin $(\mathrm{ng} / \mathrm{ml})$ & $16.52 \pm 12.46$ & $13.24 \pm 6.88$ & 0.04 \\
Vitamin B12 (pg/ml) & $352.12 \pm 124.08$ & $342.56 \pm 36.82$ & 0.64 \\
Folic acid (ng/ml) & $12.64 \pm 16.08$ & $10.94 \pm 6.16$ & 0.12 \\
$25(\mathrm{OH})$ vitamin D & $12.48 \pm 11.68$ & $9.88 \pm 4.68$ & 0.03 \\
\hline
\end{tabular}

RLS: restless leg syndrome 
RLS prevalence as $3 \%$ in CD group, which was similar with the control group and we did not determine an increased prevalence of RLS in children with CD when compared with the control cases. In our study, the population was very heterogeneous with a large range of age and moreover, the time passed from the diagnosis or the time passed with GFD was quite different among the patients. This may be the main reason why we did determine the RLS prevalence lower than previous studies and we did not determine any significant difference between Celiac patients and controls regarding the vitamin levels. Low level of education was independently associated with restless legs syndrome in the Dortmund Health Study ${ }^{17}$. In this study, we did not determine any difference regarding educational level of patients between 2 groups and for that reason; it may not affect RLS results.

To the best of our knowledge, in this study, for the first time in the literature, we have reported a negative correlation between the RLS severity and serum ferritin, folate and vitamin $\mathrm{D}$ levels, in patients with $\mathrm{CD}$. In the pathophysiological aspect, the circadian rhythm of the RLS symptoms has been associated with the circadian fluctuation of dopamine in the substantia nigra. ${ }^{18,19}$ Medications that increase the dopaminergic activity have been reported to diminish RLS symptoms. ${ }^{20}$ In that aspect, iron, which is a cofactor in dopamine production, has a central role in pathophysiology of RLS. ${ }^{21}$ The role of iron deficiency in the pathophysiology of RLS and a negative correlation of serum ferritin levels with symptom severity has been shown in previous studies. ${ }^{22,23}$ Similarly, in a recent study Cho et al. ${ }^{24}$ also reported that patients with more severe RLS symptoms had significantly lower ferritin levels. In another study, Sloand et al. ${ }^{25}$ showed that the high-dose iron dextran infusion or addition of iron supplements in some patients with anemia could reduce the severity of symptoms. However, the data about the association of iron stores with RLS in celiac patients is limited. Manchanda et al. ${ }^{26}$ suggested the investigation for CD by simple serologic tests in patients with RLS and low serum ferritin levels without an obvious cause for iron deficiency. Although, there was not a significant difference regarding serum ferritin levels between CD patients and control cases, we determined significantly lower ferritin levels in celiac patients with RLS compared with the celiac patients without RLS, supporting this data. Moreover, there was also a negative correlation between the ferritin levels and symptom severity in $\mathrm{CD}$ group encouraging the association of iron stores with the RLS pathophysiology.

In the literature, the data about folic acid and RLS is generally restricted to pregnant women. Lee et al. ${ }^{27}$ reported that compared with those without complaints of RLS, those with restless legs had significantly lower folate levels. Vahdat et al. ${ }^{28}$ determined the prevalence of RLS as $17.8 \%$ in 443 pregnant women and did not determine any significant differences between the 2 groups in terms of folate and iron supplementation or hemoglobin levels. Recently, Altunayoglu et al. ${ }^{29}$ investigated the prevalence and associated factors of RLS among a random sample of 3789 adults in our country and reported the prevalence as $4.5 \%$ and did not determine any differences in serum folic acid levels between the patients and controls. In this study, we did not determine any significant difference between $\mathrm{CD}$ patients and the control group regarding the serum folate levels but in CD group, among patients with RLS there was a negative correlation between serum folate levels and symptom severity. Folic acid therapy has been suggested to improve the symptoms of RLS which may

Table III. The Results of Correlation Analysis Performed Among CD Cases.

\begin{tabular}{lllll}
\hline & \multicolumn{2}{l}{ Age at the onset of RLS symptoms } & \multicolumn{2}{l}{ Disease severity } \\
\hline & $\mathrm{r}$ & $\mathrm{p}$ & $\mathrm{r}$ & $\mathrm{P}$ \\
Hemoglobin $(\mathrm{g} / \mathrm{dl})$ & -0.11 & 0.72 & -0.32 & 0.40 \\
Ferritin $(\mathrm{ng} / \mathrm{ml})$ & -0.29 & 0.44 & -0.74 & 0.04 \\
Vitamin B12 $(\mathrm{pg} / \mathrm{ml})$ & -0.50 & 0.08 & -0.27 & 0.48 \\
Folic acid $(\mathrm{ng} / \mathrm{ml})$ & -0.30 & 0.31 & -0.87 & 0.01 \\
$25(\mathrm{OH})$ vitamin D & -0.10 & 0.83 & -0.67 & 0.04 \\
\hline
\end{tabular}


be associated with the role of folate in the production and diurnal pattern of dopamine in the CNS. Vitamin D and its metabolites have been defined to increase and protect the dopaminergic neurons and their functions. For that reason, vitamin $\mathrm{D}$ deficiency has been suggested to play a role in RLS pathophysiology. Oran et al. ${ }^{30}$ divided adult patients who were admitted with musculoskeletal symptoms into 2 groups according to the presence of vitamin $\mathrm{D}$ deficiency and compared them for the presence of RLS. They determined a significant association between RLS and vitamin $\mathrm{D}$ deficiency. In this study, we have reported a significant negative correlation between serum $25(\mathrm{OH})$ vitamin D levels and RLS symptoms in $\mathrm{CD}$ group.

There are some limitations of this study that should be mentioned. CD patients were all under GFD which may alter the RLS symptoms and laboratory data. Our study group was very heterogeneous regarding the time passed under GFD which may be the reason of differences between our results and the previous literature. One more point is that the questionnaire performed in this study was not validated in Turkish population but the RLS was diagnosed according to the IRLSSG diagnostic criteria. Although all celiac patients included in the study were under GFD for different time periods, we did not ask any questions about the compliance of patients or we did not analyze the antibody positivity rate during the study.

In conclusion, in this study we did not determine an increased prevalence of RLS in children with CD. However, in CD group, the age at the onset of RLS symptoms was significantly younger and the disease was more severe in $C D$ group compared with the control cases. In CD patients with RLS, serum ferritin and $25(\mathrm{OH})$ vitamin $\mathrm{D}$ levels were significantly lower than those of CD patients without RLS. Moreover, there was a negative correlation between the serum ferritin, folate and vitamin D levels and symptom severity in CD group. To the best of our knowledge, this is the first study in literature evaluating the associated factors of RLS in children with CD. Larger studies with longer follow-up periods are warranted to elucidate the association of RLS with CD.

\section{REFERENCES}

1. Ludvigsson JF, LefflerDA, Bai JC, et al. The Oslo definitions for coeliac disease and related terms. Gut 2013; 62: 43-52.

2. Wierdsma NJ, van Bokhorst-de van der Schueren MA, Mulder CJ, van Bodegraven AA. Vitamin and mineral deficiencies are highly prevalent in newly diagnosed celiac disease patients. Nutrients 2013; 5: 3975-3992.

3. Hernandez L, Green PH. Extraintestinal manifestations of celiac disease. Curr Gastroenterol Rep 2006; 8: 383-389.

4. Ekbom K, Ulfberg J. Restless legs syndrome. J Intern Med 2009; 266: 419-431.

5. Yeh P, Walters AS, Tsuang JW. Restless legs syndrome: a comprehensive overview on its epidemiology, risk factors, and treatment. Sleep Breath 2012; 16: 987 1007.

6. Ohayon MM, O'Hara R, Vitiello MV. Epidemiology of restless legs syndrome: A synthesis of the literature. Sleep Med Rev 2012; 16: 283-295.

7. Innes KE, Selfe TK, Agarwal P. Prevalence of restless legs syndrome in North American and Western European populations: a systematic review. Sleep Med 2011; 12: 623-634.

8. Satija P, Ondo WG. Restless legs syndrome: pathophysiology, diagnosis and treatment. CNS Drugs 2008; 22: 497-518.

9. Earley CJ, Kuwabara H, Wong DF, et al. The dopamine transporter is decreased in the striatum of subjects with restless legs syndrome. Sleep 2011; 34: 341-347.

10. Bai JC, Fried M, Corazza GR, et al. World Gastroenterology Organization. World Gastroenterology Organisation global guidelines on celiac disease. J Clin Gastroenterol 2013; 47: 121-126.

11. Marsh MN. Gluten, major histocompatibility complex, and the small intestine. A molecular and immunobiologic approach to the spectrum of gluten sensitivity ('celiac sprue'). Gastroenterol 1992; 102: 330-354.

12. Walters AS, LeBrocq C, Dhar A, et al. International Restless Legs Syndrome Study Group. Validation of the International Restless Legs Syndrome Study Group rating scale for restless legs syndrome. Sleep Med 2003; 4: 121-132.

13. Moccia M, Pellecchia MT, Erro R, et al. Restless legs syndrome is a common feature of adult celiac disease. Mov Disord 2010; 25: 877-881.

14. Weinstock LB, Walters AS, Mullin GE, Duntley SP Celiac disease is associated with restless legs syndrome. Dig Dis Sci 2010; 55: 1667-1673.

15. Marild K, Morgenthaler TI, Somers VK, et al. Increased use of hypnotics in individuals with celiac disease: a nationwide case-control study. BMC Gastroenterol 2015; 15: 10

16. Cikrikcioglu MA, Halac G, Hursitoglu M, et al Prevalence of gluten sensitive enteropathy antibodies in restless legs syndrome. Acta Neurol Belg 2011; 111: 282-286. 
17. Szentkiralyi A, Fendrich K, Hoffmann W, Happe S, Berger K. Socio-economic risk factors for incident restless legs syndrome in the general population. J Sleep Res 2012; 21: 561-568.

18. Hagan MM, Havel PJ, Seeley RJ, et al. Cerebrospinal fluid and plasma leptin measurements: covariability with dopamine and cortisol in fasting humans. J Clin Endocrinol Metab 1999; 84: 3579-3585.

19. Einollahi B, Izadianmehr N. Restless leg syndrome: a neglected diagnosis. Nephrourol Mon 2014; 6: e22009.

20. Freeman A, Rye D, BliwiseDL, et al. Ropinirole for restless legs syndrome (RLS): an open-label and doubleblind placebo-controlled study. Neurology 2001; 56: 5.

21. Jellen LC, Lu L, Wang X, et al. Iron deficiency alters expression of dopamine-related genes in the ventral midbrain in mice. Neuroscience 2013; 252: 13-23.

22. Sun ER, Chen CA, Ho G, Earley CJ, Allen RP. Iron and the restless legs syndrome. Sleep 1998; 21: 371377.

23. Rizzo G, Manners D, Testa C, et al. Low brain iron content in idiopathic restless legs syndrome patients detected by phase imaging. Mov Disord 2013; 28 : 1886-1890

24. Cho YW, Song ML, Earley CJ, Allen RP. Prevalence and clinical characteristics of patients with restless legs syndrome with painful symptoms. Sleep Med 2015; 16: $775-778$
25. Sloand JA, Shelly MA, Feigin A, Bernstein P, Monk RD. A double blind, placebo-controlled trial of intravenous iron dextran therapy in patients with ESRD and restless legs syndrome. Am J Kidney Dis 2004; 43: 663-670.

26. Manchanda S, Davies CR, Picchietti D. Celiac disease as a possible cause for low serum ferritin in patients with restless legs syndrome. Sleep Med 2009; 10: 763-765.

27. Lee KA, Zaffke ME, Baratte-Beebe K. Restless legs syndrome and sleep disturbance during pregnancy: the role of folate and iron. J Womens Health Gend Based Med 2001; 10: 335-341.

28. Vahdat M, Sariri E, Miri S, et al. Prevalence and associated features of restless legs syndrome in a population of Iranian women during pregnancy. Int J Gynaecol Obstet 2013; 123: 46-49.

29. Altunayoglu Cakmak V, Koc B, Nuhoglu I, et al Prevalence of restless legs syndrome in Trabzon in the northeast Black Sea Region of Turkey: co-morbidities, socioeconomic factors and biochemical parameters. Neurol Res 2015; 37: 751-762.

30. Oran M, Unsal C, Albayrak Y, et al. Possible association between vitamin D deficiency and restless legs syndrome. Neuropsychiatr Dis Treat 2014; 10: 953-958. 